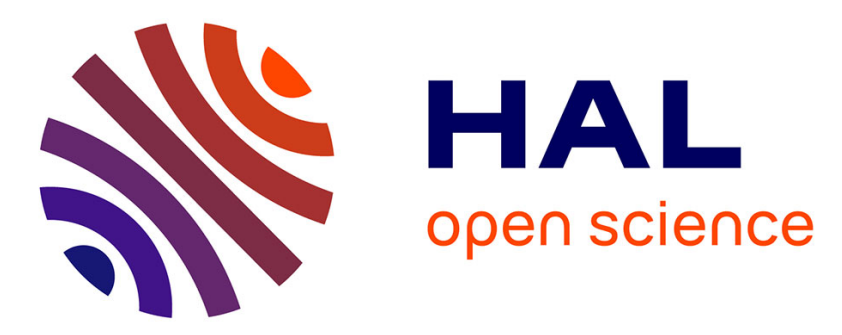

\title{
In situ experimental measurement of temperature field and surface tension during pulsed GMAW
}

Romain Monier, François Thumerel, Julien Chapuis, P Gilles, Fabien Soulié, Cyril Bordreuil

\section{- To cite this version:}

Romain Monier, François Thumerel, Julien Chapuis, P Gilles, Fabien Soulié, et al.. In situ experimental measurement of temperature field and surface tension during pulsed GMAW. Welding in the World, 2016, 60 (5), pp.1021-1028. 10.1007/s40194-016-0358-0 . hal-01576959

\section{HAL Id: hal-01576959 \\ https://hal.science/hal-01576959}

Submitted on 24 Aug 2017

HAL is a multi-disciplinary open access archive for the deposit and dissemination of scientific research documents, whether they are published or not. The documents may come from teaching and research institutions in France or abroad, or from public or private research centers.
L'archive ouverte pluridisciplinaire HAL, est destinée au dépôt et à la diffusion de documents scientifiques de niveau recherche, publiés ou non, émanant des établissements d'enseignement et de recherche français ou étrangers, des laboratoires publics ou privés. 


\title{
In situ experimental measurement of temperature field and surface tension during pulsed GMAW
}

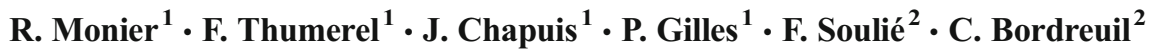

\begin{abstract}
In this paper, non-contact measurements are presented to observe droplet oscillations and to measure surface temperature during Pulsed GMAW. The techniques enable to investigate in situ the behavior of mild steel liquid metal surrounded by $\mathrm{Ar}+8 \% \mathrm{CO} 2$ plasma and then to measure tem-perature dependence of surface tension. Different tempera-tures are obtained by changing the process parameters. Surface tension values are a little higher than values found in the literature but the results demonstrate the interest of the developed setup. To conclude, a non-dimensional analysis is carried out, based on experimental results, to investigate the relative importance of the different phenomena acting during the transfer.
\end{abstract}

Keywords (IIW Thesaurus) Pulsed arc welding · GMA welding $\cdot$ Surface tension $\cdot$ Temperature $\cdot$ Steels $\cdot$ Metal transfer $\cdot$ Real time operations $\cdot$ Imaging

\section{Introduction}

The control of liquid phase is of major concern for industrial welding processes. Final bead geometry and stability of the liquid metal are driven by thermal and mass transfer, which are strongly influenced by thermophysical properties: density

R. Monier

romain.monier@areva.com

1 AREVA NP, Paris, France

2 Laboratoire de Mécanique et Génie Civil (LMGC), Université de Montpellier, Montpellier, France and viscosity. It is recognized that surface tension is one of the major properties. The surface tension depends on different parameters and plays a role along surface between liquid and shielding gas. Modeling droplet formation, understanding fluid flow in the weld pool, and predicting weld pool geometry need precise estimation of surface tension with respect to material/shielding gas composition but also with respect to temperature.

Methods to measure surface tension can be grouped in contact or non-contact methods. Because welding plasma cannot be reproduced, surface tension must be determined in situ and non-contact methods must be carried out.

Surface tension measurement method can involve a droplet levitating equipment [1] or a study of forces on a rotating pendant droplet [2]. As surface tension depends on the material but also on the environment, authors $[3,4]$ estimate surface tension of metal droplets during free fall in gas metal arc welding (GMAW) for various gas compositions. This method is based on the observation of the oscillations of the molten drop during its free fall. They used Rayleigh method [5] to study a relationship between surface tension, mass, and vibration period of a free-falling drop. Thanks to this relation and a high-speed image recording of droplets free-flight, Subramaniam [4] estimated in situ surface tension.

Because surface tension depends also on temperature, temperature measurement must also be carried out. Droplet temperature measurement has been studied through different methods, such as the heat contained in a drop with a solid state calorimeter, Soderstrom et al. [6]. To realize in situ measurements, non-contact methods are based on radiative emissions of materials, allowing a surface temperature measurement. Schopp et al. [7] and Kozakov et al. [8] determine temperature thanks to an optical emission spectrometer. They acquired spectrum during short circuit time in GMAW and during controlled arc extinction in GTAW, to avoid plasma 
radiations. To obtain the temperature, the spectral radiance is measured on a wavelength range and fitted to the Planck law with two parameters: the temperature and the emissivity. With different experiments, they obtained the emissivity as a function of temperature for S235 liquid. To obtain a surface field temperature, a high-speed camera is used to record the weld pool radiations on a specific wavelength band. With the luminance at each pixel and with the emissivity function of temperature, the temperature can be calculated. With this method, a good approximation of emissivity for given cases (gas, welding energy, and material) is obtained for wavelength between 660 and $840 \mathrm{~nm}$. According to Kozakov's study [8], weld pool temperatures lie between 1800 and $2400 \mathrm{~K}$, depending on shielding gasses. These methods need a long calibration procedure to estimate the emissivity in function of temperature. Another radiation method based on gray emissivity assumption $(\varepsilon=\mathrm{cst})$ is the two-color pyrometer. Yamasaki et al. [9] extended the punctual method of twocolor pyrometer to a two-dimensional measurement. For that, the field of view observed by a camera is optically duplicated. Between the sensor and the duplication device, each signal is filtered at a different wavelength. Finally, they obtain two times the same image filtered at two different wavelengths (950 and $980 \mathrm{~nm}$ ) on the same sensor. Temperatures are measured during the droplet-free flight with a low current $(0-40$ A). The results of average droplet surface temperature are $2280 \mathrm{~K}$ for $20 \% \mathrm{CO}_{2}$ to $2440 \mathrm{~K}$ for $100 \% \mathrm{CO}_{2}$ droplets.

In this paper, an original experimental setup is developed to estimate surface tension and temperature fields. The setup allows to investigate the correlation between surface temperature measurement and surface tension inside the plasma. In the first section, the experimental setup is described. The noncontact methods are based on the oscillations of liquid droplet for the surface tension, and two-color pyrometer of the droplet is used for the temperature measurement. In the second section, tests are done to evaluate the influence of process parameters on these two properties during pulsed GMAW. To conclude, some non-dimensional analyses based on these measurements are performed to discuss the influence of surface tension on the metal transfer.

\section{Experimental setup and methods}

\subsection{Experimental setup and GMAW settings}

The experimental technique is developed to measure in situ surface tension and temperature with non-contact devices. To observe single spherical droplet, a synergic pulsed gas metal arc welding (PGMAW) is used. PGMAW allows to select not just a single constant welding current but allows to set peak current and period as well as background current. By adjusting these parameters, droplet size and low thermal radiations of the plasma can be controlled. The droplet detachment from the wire is driven by the peak current. At the end of the peak, the droplet flights from the wire to the weld pool. During this period (low current), the liquid is always surrounded by a thermal plasma with higher optical emission in the visible range. To correlate surface tension and temperature, measurements must be carried out on the same droplet. The two noncontact imaging sensors must be synchronized on the same timebase.

The experimental system developed to realize these in situ measurements is presented in Fig. 1. It is composed of two separate parts: the two color pyrometer for temperature and a high-speed camera for droplet oscillations.

Because thermal plasma has higher optical emission in the visible range, all measurements are carried out in the nearinfrared range (850-1000 nm). For the pyrometer, the two sensors (Prosilica-Allied Vision Technology) used for temperature are identical but work at different wavelengths. These sensors are triggered with the same external device and the time of the trigger is acquired on DAQ card (PCI-6229 from national instruments). For temperature, sensors are 8 bits encoding system and have a maximum frame rate of $100 \mathrm{~Hz}$. This allows to grab only one frame per droplet flight. The external device for triggering is implemented to grab images in low current period after a preset delay depending on process parameters. Because spectral radiance wants to be measured, no additional backlighting technique can be used. The optical lens and path (beam splitter) allows to have a window of interest of $6 \times 5 \mathrm{~mm}$ on a $640 \times 480$ pix sensor. Due to optical misalignments, a simple geometrical algorithm is used to find translation, rotation, and scaling between the two images.

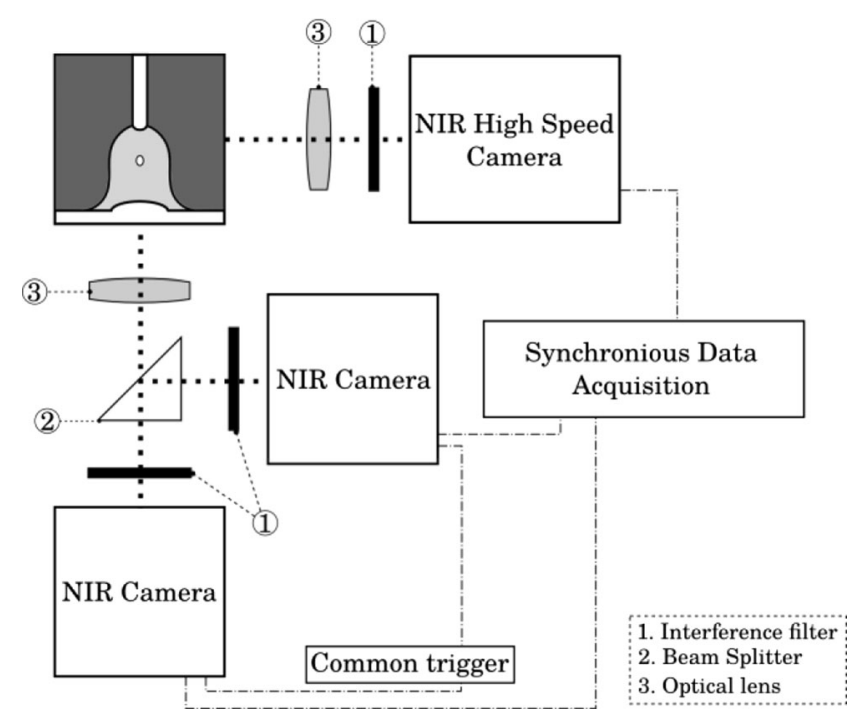

Fig. 1 Experimental device illustration, two-color pyrometer device on the lower left, and high-speed camera on the upper right 
Droplet sizes are around $1 \mathrm{~mm}$ of diameter, and oscillations are around $500 \mathrm{~Hz}$. To catch droplet oscillations, a high-speed camera working in the near-infrared (IR300-Phantom Camera) must be used. The frame rate is set between 10 and $20 \mathrm{kHz}$. A $1000-\mathrm{nm}$ narrow bandpass filter was mounted in front of the camera lens. To synchronize this camera, the end of the recording is acquired on the same DAQ card as for temperature measurement and then the time for each image is computed after the end of the experiment. The window size is around $256 \times 512$ pixels with the higher dimension corresponding to the flight direction. The resolution is then around $30 \mathrm{pix} / \mathrm{mm}$, which is enough to precisely detect oscillations.

The tests presented here are carried out with the following process parameter: spot welding (welding speed $0 \mathrm{~m} / \mathrm{min}$ ), horizontal position, S235 wire feed, S235 base material (10$\mathrm{mm}$ thick and 160-mm diameter disk). Two wire feed speeds will be used to observe the influence of process parameters. For $3 \mathrm{~m} / \mathrm{min}$, the synergic mode generates pulse of high current of $200 \mathrm{~A}$ at a frequency of $80 \mathrm{~Hz}$. Low current periods are about $12 \mathrm{~ms}$ at $30 \mathrm{~A}$. For $9 \mathrm{~m} / \mathrm{min}$, the synergic mode generates pulse of high current of $370 \mathrm{~A}$ at a frequency of $120 \mathrm{~Hz}$. Low current periods are about $9 \mathrm{~ms}$ at $80 \mathrm{~A}$.

\subsection{Temperature}

Two-color pyrometer measurements are based on material radiative emissions. The main relation to describe these emissions is Planck law. This law can be approximate through Wien's relation:

$L_{\lambda, B}(T)=\frac{C_{1} \lambda^{-5}}{e\left(C_{2} / \lambda T\right)}$

Wien's relation links the spectral radiance $L_{B}$ of the black body to the observation wavelength $\lambda$, its temperature $T$ and two constant terms $C_{1}=1.19106 \times 10^{-16} \mathrm{~W} . \mathrm{m}^{2} . \mathrm{s}$ and $C_{2}=1.4388 \times 10^{-2} \mathrm{~m} . \mathrm{K}$. This relation is valid for $\lambda T<3000 \mu \mathrm{m} . \mathrm{K}$. The definition of the emissivity $\varepsilon$ of a body is the ratio of the real body spectral radiance on the black body spectral radiance at the same temperature:

$\varepsilon_{\lambda}(T)=\frac{L_{\lambda}(T)}{L_{\lambda, B}(T)}$

With equation (1) and (2), the radiance of a real body can be expressed with:

$L_{\lambda}(T)=\varepsilon_{\lambda}(T) \frac{C_{1} \lambda^{-5}}{e\left(C_{2} / \lambda T\right)}$

In the case of two-color pyrometer measurement, the spectral radiance of the interesting zone is measured at two wavelengths $\lambda_{1}$ and $\lambda_{2}$. The ratio of the two spectral radiances can be calculated:

$\frac{L_{1}}{L_{2}}=\frac{\varepsilon_{1}}{\varepsilon_{2}} \frac{\lambda_{2}^{5}}{\lambda_{1}^{5}} e^{\left(\frac{c_{2}\left(\lambda_{1}-\lambda_{2}\right)}{T \lambda_{1} \lambda_{2}}\right)}$

The ratio of the spectral radiance is proportional to the intensity $I_{i}$ measured by the camera sensor viewed as a variation of gray level on pictures.

$\frac{L_{1}}{L_{2}}=K \frac{I_{1}}{I_{2}}$

The factor $K$ represents a correction of the difference of sensibility between the sensor of each camera at $\lambda_{1}$ and $\lambda_{2}$. This factor is calculated using spectral sensitivity curve provided by the camera constructor. With the combination of (4) and (5) and the gray body hypothesis $\left(\varepsilon_{1}=\varepsilon_{2}\right)$, the temperature can be estimated on each pixel of the camera with equation:

$T=\frac{C_{2}\left(\lambda_{1}-\lambda_{2}\right)}{\lambda_{1} \lambda_{2}} \frac{1}{\ln \left(K \frac{I_{1}}{I_{2}} \frac{\lambda_{1}^{5}}{\lambda_{2}^{5}}\right)}$

The precision of temperature estimation is directly linked to the choice of the wavelength couple. The two wavelengths must be sufficiently close to validate the gray body hypothesis but distant enough to improve the measurement precision.

The uncertainty of this method can be calculated with classical method. It is often written as follows [10]:

$\frac{\Delta T}{T}=A \cdot\left(\frac{\Delta \varepsilon_{R}}{\varepsilon_{R}}+\frac{\Delta I_{R}}{I_{R}}+\frac{\Delta K}{K}\right)$

where $\Delta \varepsilon_{R} / \varepsilon_{R}$ is the relative error associated to the gray body hypothesis, $\Delta I_{R} / I_{R}$ is the relative error on gray scale ratio and $\Delta K / K$ is the relative error on sensitivity correction coefficient, and $A$ is the governing coefficient:

$A=\left|\frac{T \lambda_{1} \lambda_{2}}{C_{2}\left(\lambda_{1}-\lambda_{2}\right)}\right|$

Wavelengths are chosen as follows: $\lambda_{1}=850 \mathrm{~nm}$ and $\lambda_{2}=$ $1000 \mathrm{~nm}$. These values are selected in a range in which arc radiations are low to minimize the error on gray scale ratio. The upper wavelength is limited to $1000 \mathrm{~nm}$ to validate Wien's hypothesis and also because of the lack of sensitivity of camera sensor over this value. Thanks to it, the value of the $A$ coefficient in case of a temperature of $2000 \mathrm{~K}$ is 0.788 . As $A$ value is under one, this wavelength choice seems to reduce uncertainty at $2000 \mathrm{~K}$. The value exceeds one for a temperature of $2539 \mathrm{~K}$.

In Fig. 2, the droplet is acquired just after detachment. The shape is almost spherical. The gray level of the left image is higher than the right one due to the sensor sensibility. In this 
Fig. 2 Frame of each camera, $\lambda_{1}$ on the left, $\lambda_{2}$ on the right during the free flight of the droplet, in the low current period. We can note the arc attenuation obtained with arc filtering
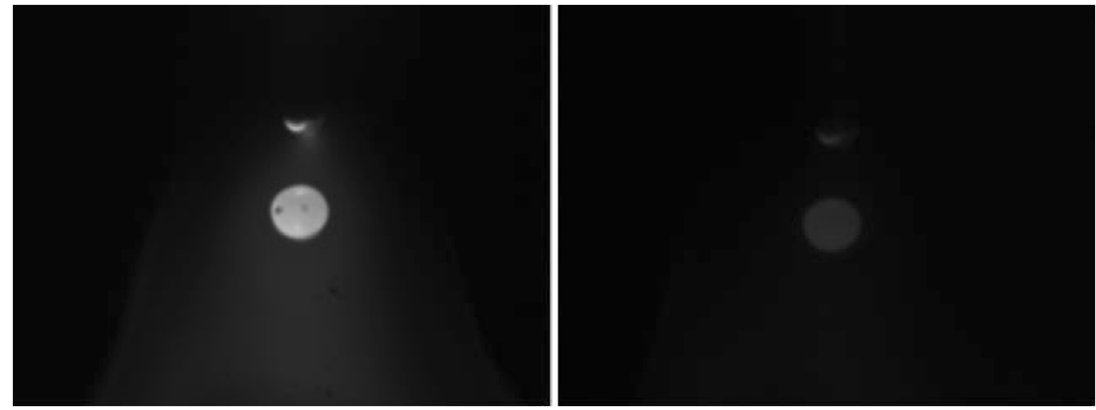

figure, it is observed low plasma emission at these wavelengths. In the left figure, the liquid at the wire tip is visible. This leads to a punctual reflection at the top of the droplet that can modify locally the temperature measurement.

Then applying (6) on each pixel of these images, surface temperatures fields are estimated. For a better readability of the results, a threshold is used to select the droplet and to delete the background on the result. Temperature fields are represented on Fig. 3, and the estimated mean temperature on the droplet is $1987 \mathrm{~K}$.

In Fig. 3, the obtained temperature field shows a quite homogeneous distribution at the surface of the droplet and the perturbation due to the reflexion of the wire tip is low. For this droplet, the temperature is higher than the melting point but is far from the evaporation point. Edge effects are mainly due to the calibration geometrical procedure.

\subsection{Droplet oscillations}

During the drop flight, surface oscillations are observable with the high speed camera. It is also observed that droplets rotate on themselves because of non-symmetric initial break-up. Depending on process parameters and for each period, droplet size can vary. To detect oscillations during the free flight period of a droplet in PGMAW, a hundred frames are available. The frame range is taken for the first frame several

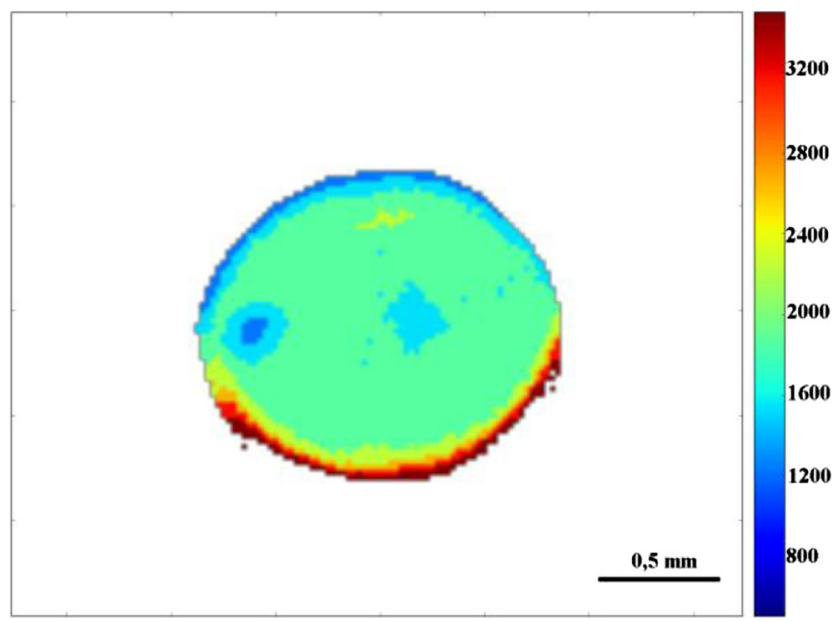

Fig. 3 Temperature fields $(K)$ of a liquid droplet milliseconds after detachment when the droplet reaches a spherical shapec and for the last one, when one part of the droplet gets out the region of interest. Droplets are chosen to have small oscillation amplitudes and small rotations during the flight.

On each frame, a contour detection algorithm is used to obtain the droplet contour as multiple points. It is based on canny numerical filter. Then, an algorithm of geometrical treatment (alpha-shape and graph theory) transforms points to segment and segments to a polygon [11]. This allows getting the virtual center of the drop and so the experimental radius $R_{\text {exp }}$ measured at each point of the contour.

Then, each contour is described through series of surface spherical harmonics with axisymmetric assumptions in which each term corresponds to one independent oscillation mode:

$\left.R(\theta, t)=R_{0} \quad 1+\sum_{l=0}^{n} a_{l}(t) P_{1}(\cos \theta)\right)$

where $R$ is the radius of the droplet in function of the angle $\theta$ from its main axis and $t$ the time of the picture. $R_{0}$ is the mean radius of the drop. It is obtained from the volume measured thanks to the hypothesis of an axisymmetric droplet. And finally, $a_{l}$ is the amplitude of the lth deformation mode linked to the Legendre polynomial $P_{l}$ of order $l$.

By comparing $R_{\text {exp }}$ and $R$, the $a_{l}$ can be evaluated thanks to a least squares method applied to each point of the contour, with the error value $\chi^{2}$ :

$\chi^{2}=\sum_{\text {points }}\left(R_{\exp }-R_{\text {legenddre }}\right)^{2}$

The description of the droplet contour with series of surface spherical harmonics fits well the reality as it is shown in Fig. 4 for one drop.

With the evaluation of the different coefficient $a_{l}$, modes deformation amplitudes can be determined with the least square minimization at each time. The evolution of parameter $a_{2}$ and $a_{3}$ is plotted in Fig. 5. Thanks to a fast Fourier transform, main frequencies of these modes are determined. In this case, the frequency values are $f_{2}=349 \mathrm{~Hz}$ for mode 2 and $f_{3}=697 \mathrm{~Hz}$ for mode 3 . 


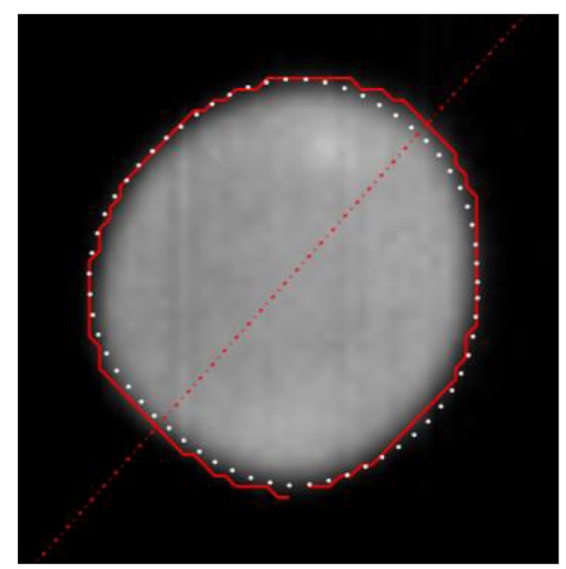

Fig. 4 Contour detection at a given time (red lines), and corresponding Legendre polynomial approximation (white dots), droplet main axis (red dots)

In Fig. 5, amplitudes are higher for mode 2 of deformation of the droplet than for mode 3 . The mode 2 is almost a sinusoidal curve but some discrepancies appear in particular at the top of the curve. For the mode 3, amplitude starts to increase at the beginning of the flight, remaining constant in the middle and then decreasing at the end. This means that it will be very difficult to measure any damping on droplet and then to measure the viscosity of the liquid metal. This evolution of amplitudes is probably due to three dimensional deformation of the free surface. Increasing the number of modes (mode 4 and 5) does not change these evolutions.

Oscillations traduce momentum balance and free surface equilibrium. These two phenomena are influenced by initial fluid velocities and physical properties. Along the free surface, normal fluid velocity is equal to the velocity of the interface. This deformation speed can be estimated from the evolution of the shape of the drop. By deriving (9) an approximation of

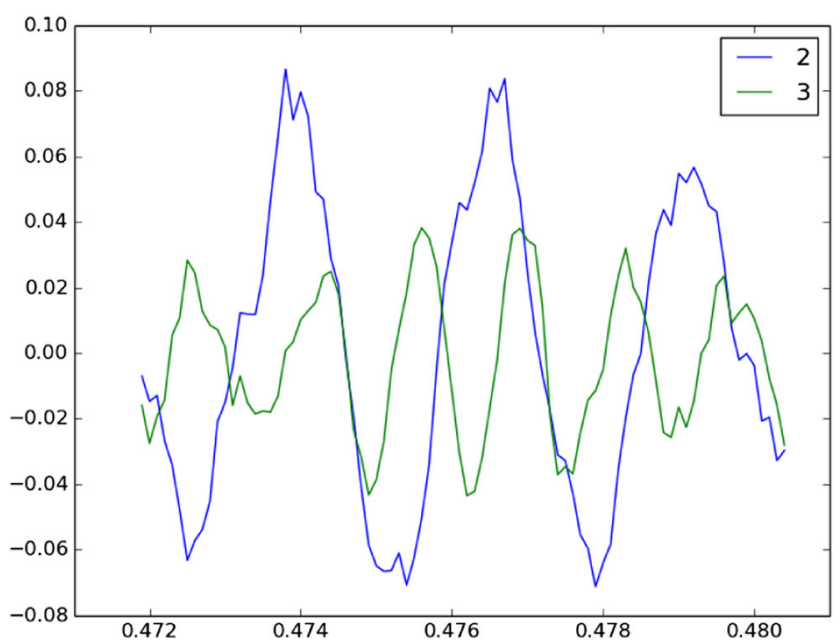

Fig. 5 Evolution of $a_{1}$ parameter as a function of time, for modes 2 and 3 overall deformation speed can be obtained. Therefore, the speed $v_{R}$ can be computed:

$v_{R}(\theta, t)=R_{0} \sum_{l=0}^{n} \frac{d a_{l}(t)}{d t} P_{l}(\cos \theta)$

The membrane deformation speed $v_{R}$ is an upper bound for internal velocities, so in a first approximation, it can be used as the worst case to compute non dimensional numbers (last section). The value of $d a_{l}(t) / d t$ is computed from the $a_{l}$ evolution curves as plotted in Fig. 5. With this method, a maximum speed of $168 \mathrm{~mm} . \mathrm{s}^{-1}$ is estimated during the flight of the drop presented here.

\subsection{Surface tension}

Surface tension is estimated thanks to the droplet oscillations. Rayleigh relation used by Subramaniam et al. [4] needs the frequency of the vibration mode in which the drop transform from an oblate to a prolate. This mode is the one with the lowest frequency. The bi-dimensional shape of the drop can be described by an infinite series of the surface spherical harmonics (9) as in Becker et al. article [11] where each term corresponds to one deformation mode. By combining Subramaniam et al. approach and Becker et al. method, the surface tension can be estimated during the droplet free-flight. The main pulsation $\Omega_{l}$ of the lth deformation mode can be estimated through Lamb [12] equation:

$\Omega_{l}^{2}=\frac{\sigma l(l-1)(l+2)}{\rho R_{0}^{3}}$

where $\sigma$ is the surface tension of the droplet and $\rho$ the metal density. By inverting (12) the surface tension can be estimated thanks to the oscillation frequency. The frequency $f_{l}$ is the one computed with the fast Fourier transformation of the evolution of the $a_{l}$ parameter during the droplet free fall. With equation (12), the mode 2 and mode 3 frequencies are within a ratio of $(30 / 8)^{1 / 2}$ (rounded to 1.93). If applied to experimental frequencies found earlier, the ratio is around 1.99. This validates for this kind of droplet, the use of equation (12) to estimate surface tension. With the definition of the pulsation $\Omega_{l}=2 \pi f_{\mathrm{i}}$, the experimental radius of the drop $R_{0}=0.69 \times 10^{-3} \mathrm{~m}$ and the density of the liquid taken from the literature $\rho=6500 \mathrm{~kg} \mathrm{~m}^{-3}$, the value of surface tension can be estimated. Here, the droplet surface tension is evaluated at $1.28 \mathrm{~N} \mathrm{~m}^{-1}$ for $l=2$ and $1.35 \mathrm{~N} \mathrm{~m}^{-1}$ for $l=3$. The main incertitude for surface tension measurement comes from the estimation of the radius and the frequency. Due to the very high frame rate, the droplet frequency uncertainty is driven by the uncertainties on the radius. For the droplet chosen, the relative error on the radius is around $2.5 \%(0.5$ pix over 20 pix) leading to a relative error on surface tension around $7.5 \%$. 


\section{Process parameters influence}

The influence of process parameters on temperature and surface tension is studied for two wire feed speeds in the same welding conditions (gas, base metal, wire feed material, transfer type) as in part 2. The two wire feed speeds are 3 and $9 \mathrm{~m} /$ min in pulsed mode, respectively. Mean powers are, respectively, 4.32 and $9.65 \mathrm{~kW}$. Due to the selection procedure of droplets (size and orientation), measurements were realized on 20 droplets for each parameter.

Concerning temperature measurements, the average surface temperatures of droplet are for the lower power parameter $(3 \mathrm{~m} / \mathrm{min}) 1822 \mathrm{~K}$ and for the $9 \mathrm{~m} / \mathrm{min}$ parameter, $2019 \mathrm{~K}$. The difference of $200 \mathrm{~K}$ on the mean value of these samples seems sufficient enough to involve it to the variation of process parameter and not to the measurement error. The difference of $200 \mathrm{~K}$ represents more than $10 \%$ of the measured temperature. The standard deviation of the $3 \mathrm{~m} / \mathrm{min}$ samples is 121 and $131 \mathrm{~K}$, respectively, for the $9 \mathrm{~m} / \mathrm{min}$ samples. These standard deviations represent $5 \%$ of the measured values. So measurement dispersion is low. As expected, with increasing welding speed and mean power, the mean temperature of droplet is higher.

The evaluation of surface tension is done on the same samples. The surface tension distribution is presented in Fig. 6.

In Fig. 6, the distribution of the values of the $9 \mathrm{~m} / \mathrm{min}$ parameter is more heterogeneous. This may be due to the more erratic detachment of the droplet at this wire speed. With higher peak current, the Lorentz forces are higher and they accelerate more the liquid at the tip of the electrode. Momentum is hardly balanced by surface tension and the droplets become more distorted. In this situation, the Rayleigh oscillation model application can be questionable.

Surface tension mean value for the $3 \mathrm{~m} / \mathrm{min}$ parameter is 1.7 and $1.5 \mathrm{~N} \mathrm{~m}^{-1}$ for the $9 \mathrm{~m} / \mathrm{min}$ parameter. The standard

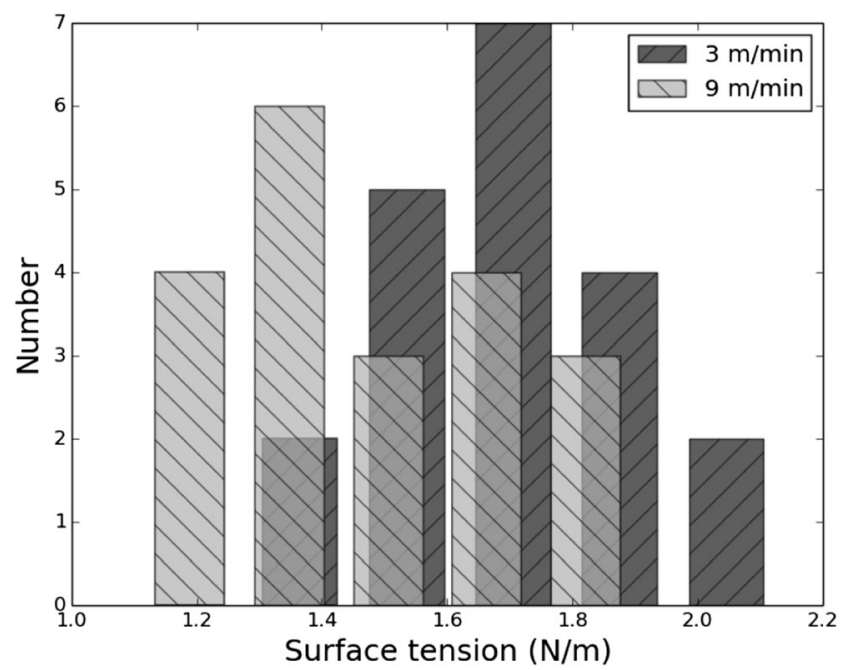

Fig. 6 Surface tension values distribution in function of process parameter: wire feed speed $3 \mathrm{~m} / \mathrm{min}$ in light grey and $9 \mathrm{~m} / \mathrm{min}$ in dark grey

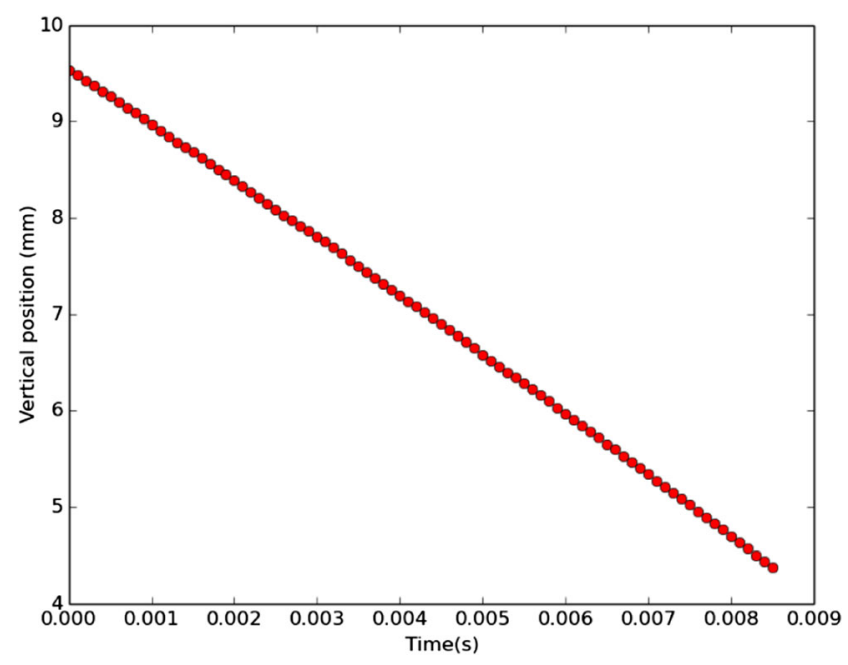

Fig. 7 Vertical position of a drop during its free flight. Constant speed

deviation value is 0.21 for $3 \mathrm{~m} / \mathrm{min}$ and 0.23 for $9 \mathrm{~m} / \mathrm{min}$. These standard deviation values are quite important. The large distribution of the values may also be due to the two dimension measurement method. The droplet shape is only described in a plane, but drops are distorted in three dimensions. So the lack of information may cause an error on oscillation frequency, and so, on surface tension estimation. Nevertheless, the observed tendency is in agreement with the mild steel behavior: the surface tension decreases when temperature increases. These estimations are done in situ during an industrial welding process and not in laboratory conditions such as magnetic levitating method. The mean values are in agreement with literature values reviewed by Subramaniam [4]. For the same configuration, mild steel and $\mathrm{Ar}+8 \% \mathrm{CO}_{2}$ shielding gas, Subramaniam measured a surface tension of $1.22 \pm 0,08 \mathrm{~N} \mathrm{~m}^{-1}$. He also reviewed surface tensions between 0.9 and $1.8 \mathrm{~N} \mathrm{~m}^{-1}$ in other literature articles.

\section{Non-dimensional analysis of droplet behavior}

From breakup to the flight, the liquid metal is subjected to complex thermal and mechanical loading. Different thermophysical properties are entering in the governing equations. Based on experimental results of the previous sections, some non-dimensional numbers are computed in order to identify the most important phenomena and the most important properties to measure.

From the position of the virtual center, droplet vertical velocity during the transfer is reported in order to have more non-dimensional numbers. Figure 7 shows the drop vertical position during its flight. The position curve is a line with a constant slope corresponding to the vertical speed of the drop. The measured speed is $600 \mathrm{~mm} \mathrm{~s}^{-1}$. No acceleration due to the gravity field is observed, the free flight assumption is validated. 


\subsection{During the free flight}

During the free flight, the droplet is loaded by gravity body forces, surface tension along the surface, viscous effects inside the bulk, and inertial forces.

Bond number, $B o$, is useful to appreciate the relative effect between gravity forces and surface tension (13):

$B o=\frac{\Delta \rho g R_{0}^{2}}{\sigma}$

With $\Delta \rho$ the difference between volumetric mass of the two fluids, $g$ the gravity acceleration, $R_{0}$ the radius measured on high-speed camera pictures $(0.69 \mathrm{~mm})$ and $\sigma=1.27 \mathrm{~N} \mathrm{~m}^{-1}$ the surface tension. Here, $B o=0.24$, so it confirms the previous assumption: gravity effect on the droplet shape is negligible during its free flight.

Ohnesorge number $\mathrm{Oh}$ is the ratio between viscous forces and surface tension coupled to inertia (14):

$o h=\frac{\mu}{\sqrt{\sigma \rho R_{0}}}$

where $\mu=6.10^{-3} \mathrm{~Pa}$ s is the dynamic viscosity of the mild steel used, $\sigma=1.27 \mathrm{~N} \mathrm{~m}^{-1}$ and $R_{0}=0.69 \mathrm{~mm}$. Applying (14) to the studied drop gives a result of $8.10^{-5}$. The oscillation model used here has been discussed by A. Prosperetti [13]. It shows that viscous effects become negligible if the condition $O h<0.1$ is respected. The value of the experiment presented here fulfills this condition. So viscous effects are negligible on droplet deformation.

The Weber number indicates the drop tendency to split itself in other smaller drops. It is the relation between inertia forces and surface tension (15):

$W e=\frac{\rho v_{R}^{2} R_{0}}{\sigma}$

In our configuration, the obtained Weber number is $W e=0.1$. Its critical value is 12 . So, the estimated Weber of 0.1 indicates that inertial forces would not split the drop, surface tension effort is predominant and ensures the droplet integrity. This value also means that initial velocities induced by pinch effect do not prevail on surface tension.

\subsection{During the detachment}

The gravity bond number and the Ohnesorge number are not changing for this configuration.

During the breakup, the fluid phase at the tip of the wire is mainly loaded by Lorentz force. The detachment is occurring during the peak (around $200 \mathrm{~A}$ ). The low current is around $30 \mathrm{~A}$. To see the influence of current, the magnetic Bond number is computed:

$M=\frac{\mu_{0} I^{2}}{4 \pi^{2} R_{0} \sigma}$

$\mu_{0}$ is the permeability equals $4 \pi 10^{-7} \mathrm{~A} \mathrm{~m}^{-2}$. With these data, the magnetic bond number is around 4 in the peak current and 0.04 in the low current denoting the relatively high effect of Lorentz during the peak current with respect to tension surface.

Just after the droplet detachment, the velocity is measured around $600 \mathrm{~mm} / \mathrm{s}$. In this condition, the Weber number can be computed in order to see the relative influence of inertial effects against surface tension effects. The Weber is around 2 denoting the increasing of initial effect but it can be still be neglected.

All these indicators underline the importance of surface tension in the behavior of liquid metal droplet and so the necessity of in situ measurement for a better knowledge of welding process.

\section{Conclusion}

The in situ measurements realized in this study show results in accordance of literature review. The coupled devices enable synchronous measurements of surface temperature fields and surface tension and seem to not be yet reported in the literature.

Actually, surface temperature estimation gives underestimated results but qualitative comparison can still be done. Temperature measurement device needs a precise calibration process to decrease uncertainties on the controlled parameters such as $K$ the correction coefficient. Thus, the last source of error would only be the uncertainty induces by the lack of knowledge on emissivity values. This can be reduced with a clever wavelengths choice. This part of the device has to be studied on a reference situation to perform an evaluation of its accuracy.

On the other hand, surface tension estimation thanks to Rayleigh equation gives results really close to literature values. This estimation can be done if the drop is not too much disturbed. So future work should be oriented to the evaluation of high deformation droplets surface tension.

To conclude, thanks to this device, some parametric study could link in situ surface tension to temperature in different process parameters. And so evaluate the influence of different welding parameters such as shielding gas, materials but also energies on these important physical properties.

\section{References}

1. Rhim WK, Ohsaka K, Paradis PF, Spjut RE (1999) Noncontact technique for measuring surface tension and viscosity of molten materials using high temperature electrostatic levitation. Rev Sci Instrum 70(6):2796-2801 
2. Hansen FK, Rødsrud G (1991) Surface tension by pendant drop: I. A fast standard instrument using computer image analysis. J Colloid Interface Sci 141(1):1-9

3. Bachmann B, Siewert E, Schein J (2012) In Situ droplet surface tension and viscosity measurements in gas metal arc welding, $\mathrm{J}$ Phys D Appl Phys Vol 45

4. Subramaniam S, White DR (2001) Effect of shield gas composition on surface tension of steel droplets in a gas-metal-arc welding arc. Metall Mater Trans B 32(2):313-318

5. Lord R (1879) Proc R Soc Lond XXIX:71-97

6. Soderstrom EJ, Scott KM, Mendez PF (2011) Calorimetric measurement of droplet temperature in GMAW. Weld J 90(4):1s-8s

7. Schöpp H, Sperl A, Kozakov R, Gött G, Uhrlandt D, \& Wilhelm G "'Temperature and emissivity determination of liquid steel S235". J Phys D Appl Phys 45, Issue 23, 235203
8. Kozakov R, Schöpp H, Gött G, Sperl A, Wilhelm G, Uhrlandt D (2013) Weld pool temperatures of steel S235 while applying a controlled short-circuit gas metal arc welding process and various shielding gases". J Phys D Appl Phys 46(47):475501

9. Yamazaki K, Yamamoto E, Suzuki K, Koshiishi F, Waki K, Tashiro S, Nakata K (2010) The measurement of metal droplet temperature in GMA welding by infrared two-colour pyrometry. Weld Int 24(2):81-87

10. Thevenet J, Siroux M, Desmet B (2010) Measurements of brake disc surface temperature and emissivity by two-color pyrometry. Appl Therm Eng 30(6):753-759

11. Romero E, Chapuis J, Soulié FC, Bordreuil GF (2013) Image processing and geometrical analysis for profile detection during pulsed gas metal arc welding. Proc Inst Mech Eng B J Eng Manuf 227(3):396-406

12. Lamb H (1932) Hydrodynamics. Cambridge university press

13. Prosperetti A (1980) Free oscillations of drops and bubbles, the initial-value problem. J Fluid Mech 100:333-347 